\title{
Interaction of Natural Compounds in Licorice and Turmeric with HIV-NCp7 Zinc Finger Domain: Potential Relevance to the Mechanism of Antiviral Activity
}

\author{
Runjing Wang ${ }^{1,2,+}$, Yinyu Wei ${ }^{1,+}$, Meiqin Wang ${ }^{1}$, Pan Yan ${ }^{1}$, Hongliang Jiang ${ }^{1}$ and Zhifeng Du ${ }^{1, *}$ \\ 1 Tongji School of Pharmacy, Huazhong University of Science and Technology, Wuhan 430030, China; \\ wangrjcn@foxmail.com (R.W.); weinyinyu@163.com (Y.W.); wangmeiqin@hust.edu.cn (M.W.); \\ panyan_science@163.com (P.Y.); jianghongliang@hust.edu.cn (H.J.) \\ 2 Ningxia Pharmaceutical Inspection and Research Institute, Yinchuan 750001, China \\ * Correspondence: duzhifeng@hust.edu.cn \\ + These authors contributed equally to this work.
}

\section{check for} updates

Citation: Wang, R.; Wei, Y.; Wang, M.; Yan, P.; Jiang, H.; Du, Z. Interaction of Natural Compounds in Licorice and Turmeric with HIV-NCp7 Zinc Finger Domain:

Potential Relevance to the Mechanism of Antiviral Activity. Molecules 2021, 26, 3563. https://doi.org/10.3390/ molecules26123563

Academic Editor: Kyoko

Nakagawa-Goto

Received: 7 April 2021

Accepted: 9 June 2021

Published: 10 June 2021

Publisher's Note: MDPI stays neutral with regard to jurisdictional claims in published maps and institutional affiliations.

Copyright: (c) 2021 by the authors. Licensee MDPI, Basel, Switzerland. This article is an open access article distributed under the terms and conditions of the Creative Commons Attribution (CC BY) license (https:// creativecommons.org/licenses/by/ $4.0 /)$.
Abstract: Nucleocapsid proteins (NCp) are zinc finger (ZF) proteins, and they play a central role in HIV virus replication, mainly by interacting with nucleic acids. Therefore, they are potential targets for anti-HIV therapy. Natural products have been shown to be able to inhibit HIV, such as turmeric and licorice, which is widely used in traditional Chinese medicine. Liquiritin (LQ), isoliquiritin (ILQ), glycyrrhizic acid (GL), glycyrrhetinic acid (GA) and curcumin (CUR), which were the major active components, were herein chosen to study their interactions with HIV-NCp7 C-terminal zinc finger, aiming to find the potential active compounds and reveal the mechanism involved. The stacking interaction between NCp7 tryptophan and natural compounds was evaluated by fluorescence. To elucidate the binding mode, mass spectrometry was used to characterize the reaction mixture between zinc finger proteins and active compounds. Subsequently, circular dichroism (CD) spectroscopy and molecular docking were used to validate and reveal the binding mode from a structural perspective. The results showed that ILQ has the strongest binding ability among the tested compounds, followed by curcumin, and the interaction between ILQ and the NCp7 zinc finger peptide was mediated by a noncovalent interaction. This study provided a scientific basis for the antiviral activity of turmeric and licorice.

Keywords: zinc fingers; fluorescence; mass spectrometry; anti-HIV; natural product

\section{Introduction}

Zinc finger proteins are proteins that use zinc as the coordination center, and the coordination is between zinc and cysteine or histidine from peptides [1]. The nucleocapsid protein, a zinc finger protein, is particularly important, as it is highly conserved and plays a central role in HIV virus replication, mainly by interacting with nucleic acids. Therefore, it is one of the drug targets for HIV therapy [2-4], and it is complementary to drugs such as protease inhibitors and nucleoside analogs aimed at other targets of the viral life cycle [4], which cause drug resistance easily [5-7]. The presence of the W37 residue in the nucleocapsid protein has been revealed as a key feature for molecular recognition from the zinc finger toward oligonucleotides $[8,9]$.

Natural products have been shown to be able to inhibit HIV $[6,10,11]$. An example is licorice, the roots and rhizomes of Glycyrrhiza uralensis, Glycyrrhiza inflata and Glycyrrhiza glabra [12,13]. Glycyrrhizin (GL), the aqueous extract from licorice root, is effective in preventing the progression of disease in a mouse model [14,15]. The treatment comprised of GL, glycine and cysteine was found to be effective in the treatment of HIV-infected patients, as shown by the improvement in the clinical symptoms and immunological and liver functions [16]. Curcumin (CUR), a natural phytochemical derived from turmeric, also 
showed HIV inhibition activity [17-21]. Curcumin has no toxicity, and it was in clinical trials for AIDS patients [22]. The detailed mechanism underlying this is still unclear.

Small molecules from natural products can form covalent or noncovalent complexes with targets, accompanied by inhibiting the activity of enzymes or proteins, which is typically the action mode of natural compounds [23-25]. Spectrometric and spectroscopic methods are useful in revealing the interactions between small molecules and their targets [26-29]. As reported previously [6], nordihydroguaiaretic acid was found to inhibit nucleocapsid (NC) and the replication of wild-type and drug-resistant HIV-1 strains in the low micromolar range with moderate cytotoxicity. The mechanism of action was elucidated by nuclear magnetic resonance, mass spectrometry, fluorescence spectroscopy and molecular modeling. Nordihydroguaiaretic acid was found to act through a dual mechanism of action never highlighted before for NC inhibitors (NCIs) — namely, noncovalent bindingresulting in the inhibition of the nucleic acid chaperone properties of $\mathrm{NC}$, followed by the chemical oxidation of nordihydroguaiaretic acid inducing the inactivation of the protein.

Tryptophan (Trp) of the HIV-NCp7 zinc finger has a fluorescence emission, while the stacking interaction quenches that fluorescence. A previous study suggested a good correlation between tryptophan quenching and the inhibition of NCp7-nucleic acid binding [30]. Therefore, natural compounds CUR, liquiritin (LQ), isoliquiritin (ILQ), glycyrrhizic acid (GL) and glycyrrhetinic acid (GA), the major components of turmeric and licorice, were selected in this study, and the binding between the tryptophan of $\mathrm{NCp} 7$ and natural compounds was evaluated by fluorescence. The binding mode between the inhibitors and targets was studied by mass spectrometry. CD spectroscopy and molecular docking were used to validate and reveal the binding mode from a structural perspective. The chemical structures of CUR, LQ, ILQ, GL and GA are shown in Figure 1.
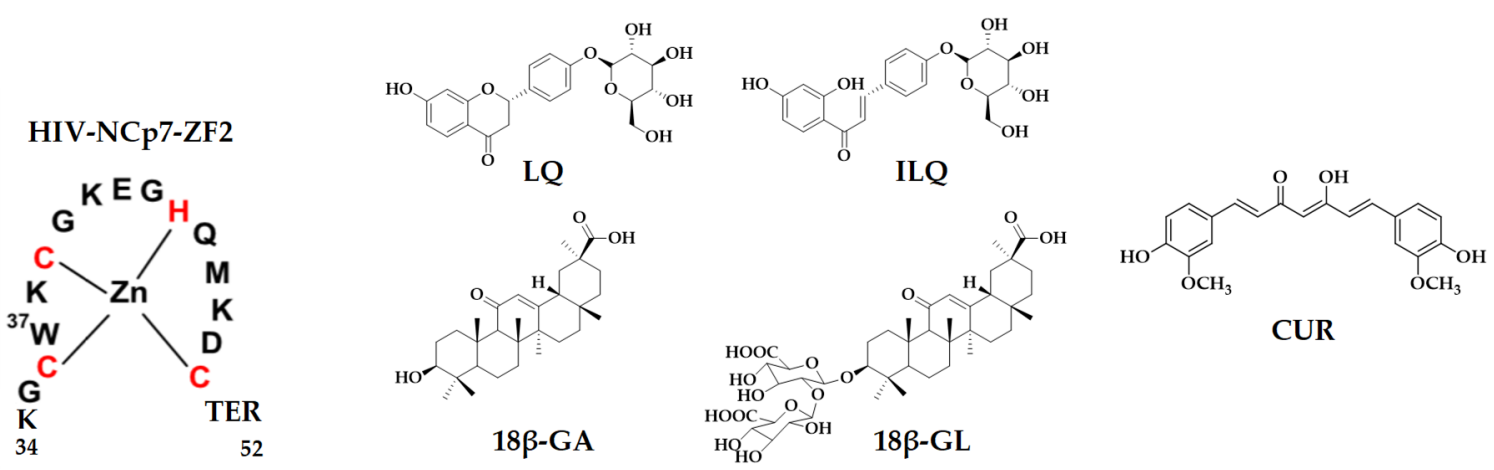

Figure 1. Sequence of the C-terminal zinc finger of HIV-NCp7 and the chemical structures of the compounds used in this study.

\section{Results and Discussion}

\subsection{Characterization of Zinc Finger by CD Spectroscopy}

Circular dichroism gives information about the secondary structure of the proteins. It is a useful tool to characterize the formation of a zinc finger, as the CD spectrum of $\mathrm{NCp} 7$ displays a positive maximum around $220 \mathrm{~nm}$. As we can see from Figure 2, the profiles of the apopeptide and zinc finger (ZF) peptide are different, according to the literature [31]. Based on the CD spectrum, the formation of the zinc finger structure was confirmed.

\subsection{Evaluation of the Binding between NCp7 ZF and Natural Compounds by Fluorescence Assay}

A library of approximately 2000 small molecules from the NCI diversity set suggested a good correlation between tryptophan quenching and the inhibition of NCp7-nucleic acid binding for fluorescein-based compounds [30]. Therefore, we evaluated the NCp7 inhibitors from natural products by fluorescence. The fluorescence quenching of Trp was measured in the presence of natural compounds in order to compare the association affinity [32]. It can be a good indicator of the strength for an interaction [33]. 


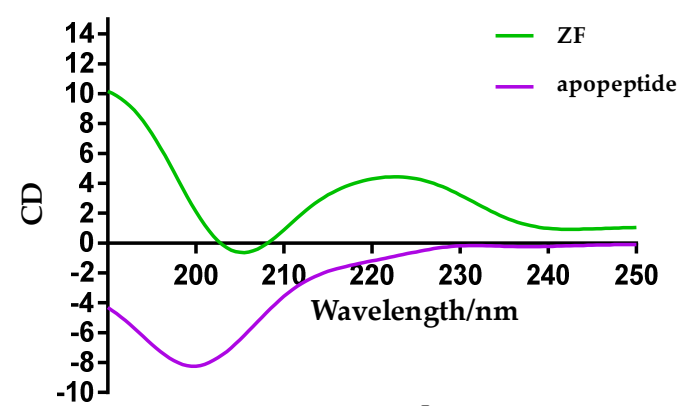

Figure 2. CD spectra of $100-\mu \mathrm{M} Z \mathrm{ZF}$ and $100-\mu \mathrm{M}$ apopeptide.

Firstly, the fluorescence of LQ, ILQ, $18 \beta$-GA and $18 \beta-$-GL and CUR was measured to exclude interference. A 5-mM concentration was prepared in DMSO. As shown in Figure S1, ILQ, GL, GA and CUR had no fluorescence under the tested conditions, while LQ had observable fluorescence. Therefore, the fluorescence of most compounds will not interfere with the measurements, except for LQ.

Subsequently, the emission spectrum of $5-\mu \mathrm{M}$ zinc finger at $\mathrm{pH} 7.4(50-\mathrm{mM} \mathrm{NaCl}$ in $20-\mathrm{mM}$ tris buffer) was acquired. The emission spectrum of $5-\mu \mathrm{M}$ zinc finger in a buffer with DMSO was also acquired to exclude the influence of an organic solvent. As we can see from Figure 3A, the DMSO has no influence on the assay. The emission spectrum of the buffer with DMSO was also acquired to exclude the fluorescence from the background. As we can see from Figure 3A, the buffer with DMSO has no fluorescence. Therefore, the DMSO and buffer will not influence the fluorescence of zinc finger during the assay.

A

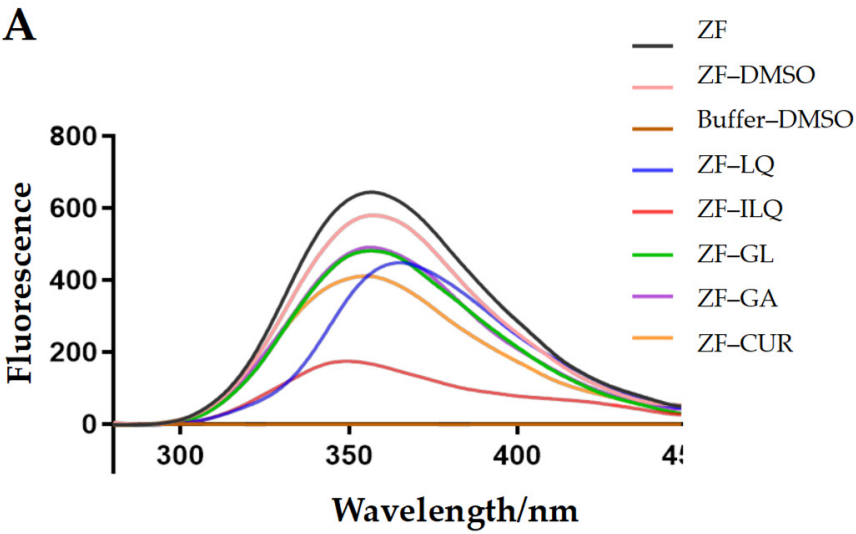

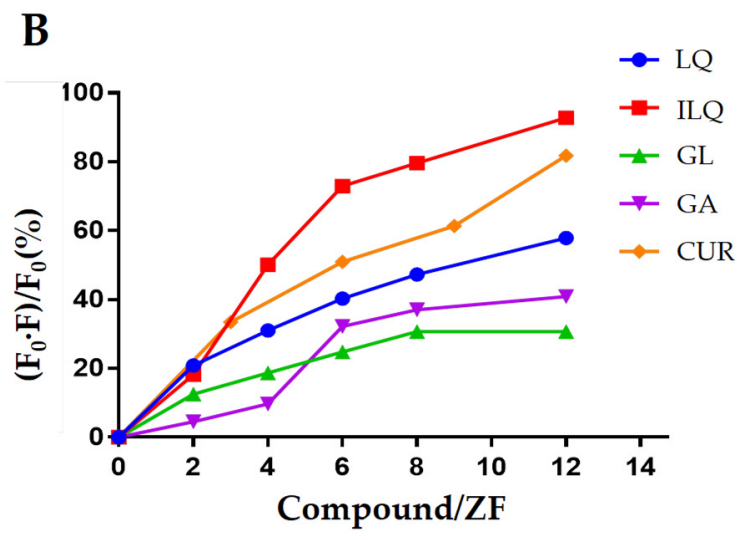

Figure 3. (A) The fluorescence emission spectra of ZF upon incubation with different natural compounds. Spectra were recorded for $5-\mu \mathrm{M}$ ZF after incubation with $30-\mu \mathrm{M}$ complexes. (B) Fluorescence quenching ratio of ZF upon adding different ratios of natural compounds ( $\mathrm{ZF}) /($ compounds)). Titration was performed with $5-\mu \mathrm{M} Z \mathrm{ZF}$ at RT. F represents the fluorescence intensity of ZF at $356 \mathrm{~nm}$ during titration, and F0 is the fluorescence intensity of ZF only at $356 \mathrm{~nm}$.

Then, the emission spectrum of 5- $\mu \mathrm{M}$ zinc finger incubated with $30-\mu \mathrm{M}$ compounds was acquired. As we can see from Figure 3A, under the same molar ratio, all the compounds can quench the fluorescence of zinc finger to some extent. The strongest quenching comes from ILQ, then CUR, and the same extent of quenching was observed for GL and GA. LQ has a similar extent of quenching; however, its own fluorescence interferes with the measurement of quenching, as we can observe by the shift of the emission spectrum.

To compare the binding strengths between the natural compounds and zinc finger, $5-\mu \mathrm{M}$ zinc finger was titrated with ILQ, LQ, GL, GA and CUR at molar ratios (ZF/compounds) from 1 to 12 . The emission maximum $(356 \mathrm{~nm})$ was measured immediately after each titration. As we can see from Figure S2 and Figure 3B, the extent of quenching was consistent with that of 6:1 for different compounds. At 12 to 1, ILQ and CUR quenched most of the fluorescence. 
Therefore, they were screened as the strongest binders among the tested compounds, which was consistent with the in vitro anti-HIV activity of the ILQ analog and CUR [20,34].

Subsequently, the binding kinetics between ZF and CUR/ILQ were studied. The fluorescence of ZF quenched by six equivalents of CUR/ILQ was measured at different time points. As shown in Figure S3, the binding was immediate, and the fluorescence remained constant even after $12 \mathrm{~h}$; therefore, we proposed the noncovalent binding mode for those two compounds with zinc finger. To validate the binding mode of ILQ with zinc finger, mass spectrometry and CD spectroscopy were utilized.

\subsection{Elucidation of the Binding Mode between NCp7 ZF and Natural Compounds by Mass Spectrometry}

Mass spectrometry has been successfully used to study the noncovalent and covalent interactions between the zinc finger and small molecules $[2,6,31,35,36]$. In this work, the reaction mixture of ZF and the active compounds was characterized by electrospray ionization-mass spectrometry (ESI-MS). Firstly, ILQ was dissolved in methanol with a concentration of $5 \mathrm{mM}$; then, it was incubated with the zinc finger peptide at the 1:3 and 1:6 molar ratios, respectively. The reaction mixture was diluted with methanol water to obtain a final concentration of $10 \mu \mathrm{M}$; subsequently, an ESI-MS analysis was carried out to monitor the reaction. For the 1:3 molar ratio, the reaction mixture was monitored at $15 \mathrm{~min}$, $2 \mathrm{~h}$ and $5 \mathrm{~h}$, respectively.

The mass spectra of the reaction mixture of the zinc finger and ILQ are shown in Figure 4, and the species at the 1:3 or 1:6 molar ratios were similar when the reaction time was $15 \mathrm{~min}$. The zinc finger was observed as the major species, and the noncovalent adduct was the minor species. The disagreement between strong fluorescence quenching and a low ratio of noncovalent adduct was due to the dissociation of the noncovalent adduct during the mass spectrometric analysis. The observation of noncovalent species and free zinc finger indicates the noncovalent binding mode between ILQ and the zinc finger at the early time points. Similarly, we observed noncovalent-binding species for CUR, as shown in Figures S6 and S8.
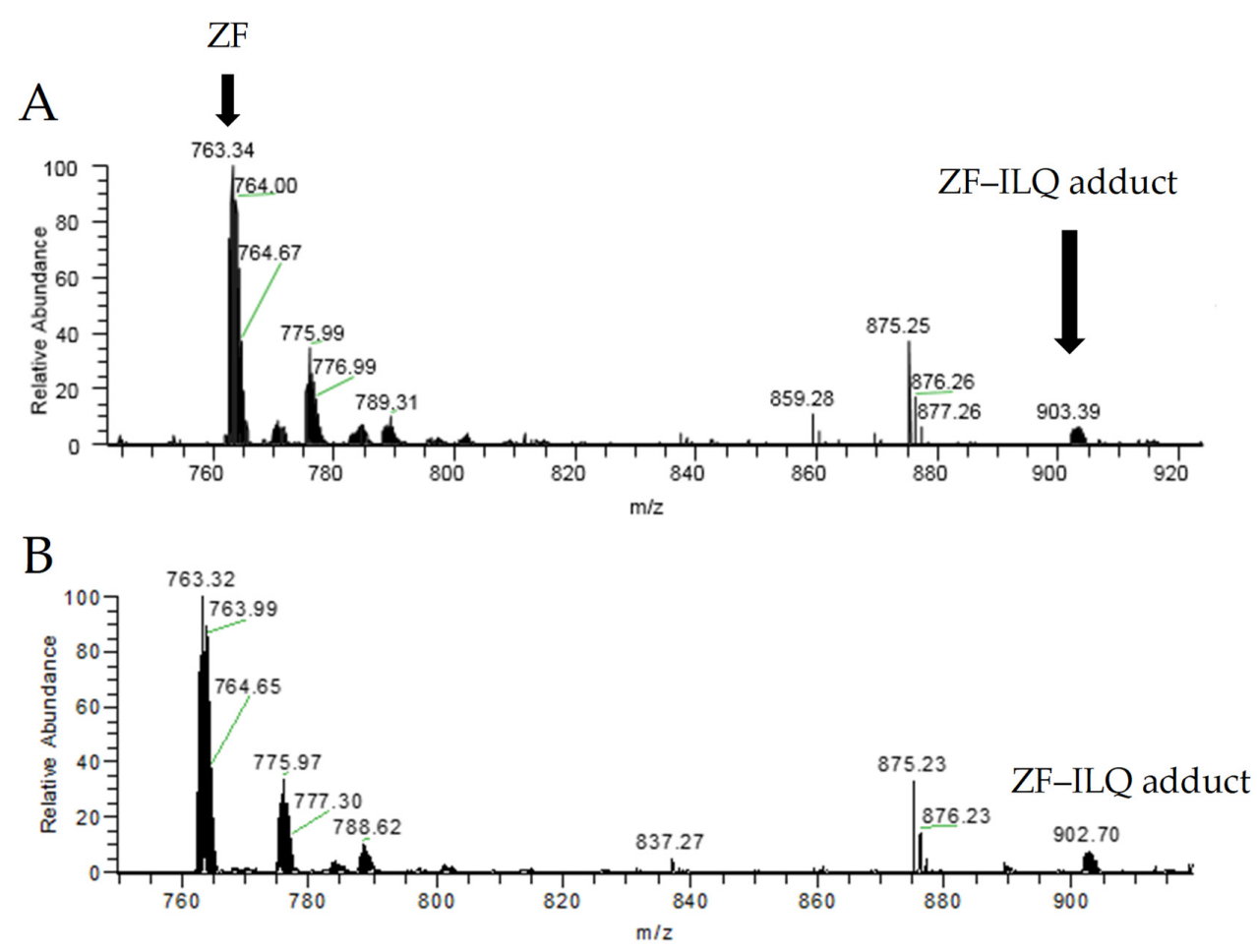

Figure 4. ESI-MS spectra of the reaction mixture between ZF and ILQ. (A) $10 \mu \mathrm{M}$ of zinc finger were incubated with $30-\mu \mathrm{M}$ ILQ for $15 \mathrm{~min}$. (B) $10 \mu \mathrm{M}$ of zinc finger were incubated with $60-\mu \mathrm{M}$ ILQ for $15 \mathrm{~min}$. 
To monitor the species formed at different time points, the reaction mixture was analyzed by ESI-MS at 1:3 for $15 \mathrm{~min}, 2 \mathrm{~h}$ and $5 \mathrm{~h}$, respectively. As shown in Figure S4, the species formed at different time points are the same, and the relative intensities of the important species are similar. These results were consistent with the kinetic results from the fluorescence analysis. Therefore, the immediate noncovalent binding mode between ILQ and zinc finger was confirmed. Similar results were observed for CUR, as shown in Figure S7.

The species observed were identified by high-resolution mass spectrometry. The theoretical and experimental spectra are shown in Figures S5 and S8, and the $m / z$ and isotope pattern are consistent between the theoretical and experimental results. The observed species are summarized in Table 1. The major species are zinc finger and the noncovalent adduct of zinc finger with ILQ, which is also the case for CUR, as shown in Table 1. At the same time, a covalent adduct of an oxidized peptide with ILQ was also observed as a minor species, while the covalent species was not observed for CUR. These results showed that noncovalent recognition will gradually induce covalent binding.

Table 1. Species observed in the ESI-MS spectra (charge +3).

\begin{tabular}{ccc}
\hline Species & Formula & Mono Observed $\boldsymbol{m} / \boldsymbol{z}$ \\
\hline ZF & $\mathrm{C}_{90} \mathrm{H}_{144} \mathrm{~N}_{30} \mathrm{O}_{28} \mathrm{~S}_{4} \mathrm{Zn}$ & 762.62 \\
ZF-ILQ adduct & $\mathrm{C}_{111} \mathrm{H}_{166} \mathrm{~N}_{30} \mathrm{O}_{37} \mathrm{~S}_{4} \mathrm{Zn}$ & 902.02 \\
Oxipeptide-ILQ & $\mathrm{C}_{111} \mathrm{H}_{166} \mathrm{~N}_{30} \mathrm{O}_{37} \mathrm{~S}_{4}$ & 880.71 \\
ZF-CUR adduct & $\mathrm{C}_{111} \mathrm{H}_{164} \mathrm{~N}_{30} \mathrm{O}_{37} \mathrm{~S}_{4} \mathrm{Zn}$ & 885.35 \\
\hline
\end{tabular}

Thereafter, we suggested that the possible mechanism was as follows: firstly, the molecule recognizes and binds NC noncovalently; in the second step, the ejection of zinc from the noncovalent adduct deprotects the cysteine amino acid in zinc finger; in the final step, the oxidation of cysteine is induced by the unsaturated keto group of ILQ. For CUR, its activity is lower than that of ILQ; thus, we did not observe the dissociation of $\mathrm{Zn}$. The inhibiting activity of ILQ towards the binding between the zinc finger peptide and nuclear acid should be tested in the future.

In summary, based on the mass spectrometric results, it is clear that ILQ initially binds to the NCp7 C-terminal zinc finger peptide by the noncovalent mode, followed by covalent binding. The possible mechanism was elucidated herein.

\subsection{Structural Information of the Binding from CD Spectroscopy and Molecular Docking}

Circular dichroism spectroscopy has been widely used to study the disturbance of zinc finger protein conformation by small molecules $[2,31,35]$. The CD spectrum of the C-terminal ZF of HIV-NCp7 is characterized by a positive band at around $220 \mathrm{~nm}$ and a negative band at 195-200 nm [31,37]. The loss of zinc from the ZF results in a decrease in the positive band and a significant increase in the intensity of the negative band. As shown in Figure 5, the CD profiles of the zinc finger protein and reaction mixture are similar. Incubation with CUR or ILQ for $2 \mathrm{~h}$ does not change the secondary structure of ZF. Therefore, the noncovalent binding mode was also validated by CD spectra.

To obtain the structural details of noncovalent binding, molecular docking was performed for the strongest binder. AutoDock is an automated docking tool. It is designed to predict how small molecules, such as substrates or drug candidates, bind to a receptor of a known 3D structure. AutoDock Vina software was used herein to show the structure of the complex. As shown in Figure 6, molecular docking revealed edge-to-face $\pi-\pi$ stacking between the protein tryptophan and ILQ aromatic ring, which was consistent with the interaction mode of the nucleic acid bases and other aromatic compounds $[2,6,32,38]$. For CUR, face-to-face $\pi-\pi$ stacking was observed between the protein tryptophan and CUR aromatic ring. The overview and zoom in for the molecule docking of HIV-NCp7 ZF with ILQ and CUR are displayed in Figure $6 \mathrm{~A}, \mathrm{~B}, \mathrm{D}, \mathrm{E}$, respectively. To give a clear view of the binding, the surface of HIV-NCp7 is displayed with ILQ and CUR attached in Figure 6C,F, respectively. 
A

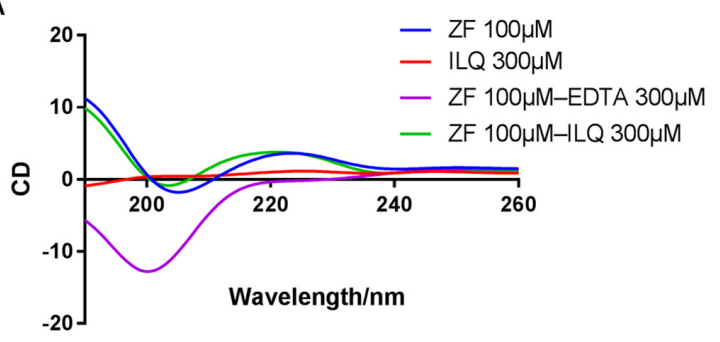

B

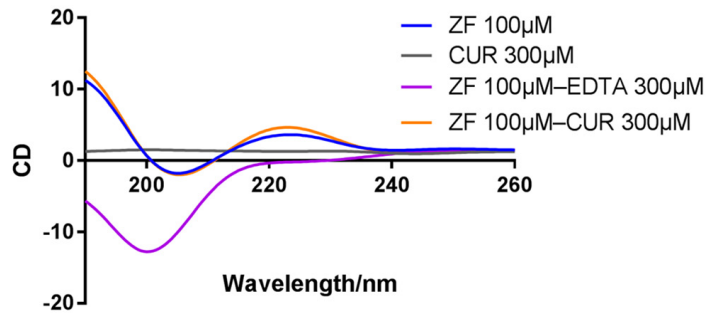

Figure 5. Characterization of the ZF structure upon ILQ or CUR binding. (A) CD spectra of ZF at $\mathrm{t}=2 \mathrm{~h}$ from the incubation with ILQ at 3:1 ([ILQ]/[ZF]) (B) CD spectra of ZF after the incubation with CUR for $2 \mathrm{~h}$ at 3:1 ([CUR]/[ZF]).

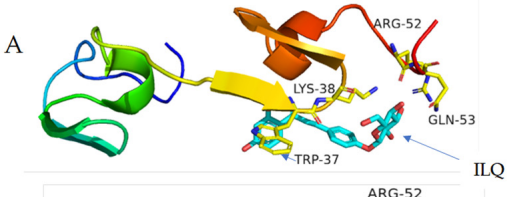

$\mathrm{D}$

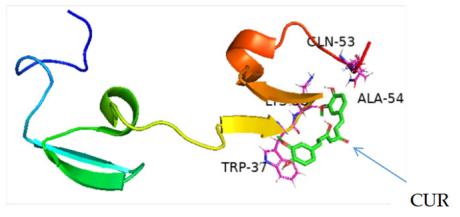

B
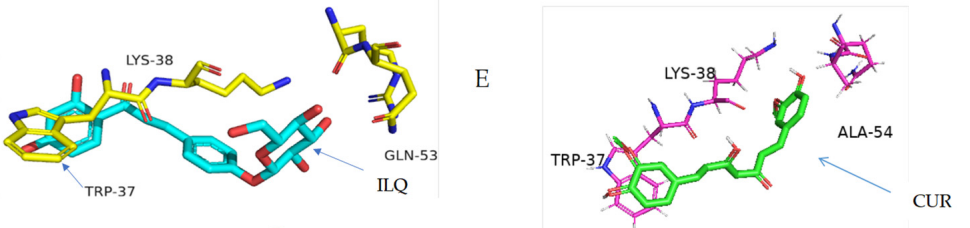

C

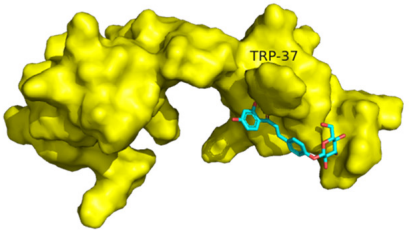

F

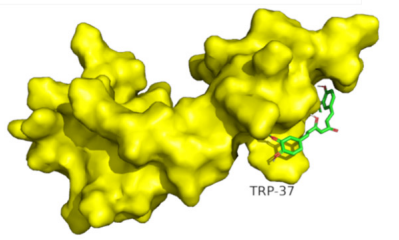

Figure 6. Molecular docking of HIV-NCp7 ZF with ILQ and CUR: (A,D) overview, (B,E) zoom in of the stacking region and $(\mathbf{C}, \mathbf{F})$ surface of the protein with ILQ and CUR, respectively.

\section{Materials and Methods}

\subsection{Reagents and Equipment}

The pure compounds of LQ, ILQ, GL, GA and curcumin were bought from the National Pharmaceutical Engineering Center for Solid Preparations of Chinese Herbal Medicine (Nanchang, China). The NCp7 C-terminal peptide sequence (KGCWKCGKEGH QMKDCTER) was purchased from China peptide Co., Ltd. (Shanghai, China). All the other reagents were purchased from Sigma Aldrich, USA and used without further purification.

Fluorescence studies were recorded on a PerkinElmer fluorometer. CD spectra were obtained with a JASCO J-1500 Spectropolarimeter (Jasco Corp., Tokyo, Japan) in the wavelength range of $190-250 \mathrm{~nm}$, using a cuvette with a path length of $0.1 \mathrm{~cm}$. Mass spectrometry experiments were carried out on an Orbitrap Velos from the Thermo Electron Corporation (Waltham, MA, USA).

\subsection{Methods and Procedure}

\subsubsection{Preparation of Zinc Finger Protein}

The preparation of the zinc finger protein followed the published methods [31]. The apopeptide was dissolved in deionized water at a concentration of $1 \mathrm{mM}$. Zinc acetate (1.2 eq.) was added to the solution, and the $\mathrm{pH}$ was adjusted to 7.0 using $\mathrm{NH}_{3} \cdot \mathrm{H}_{2} \mathrm{O}$. The zinc finger solution was incubated for $2 \mathrm{~h}$ at $37^{\circ} \mathrm{C}$ before recording any experiments. Secondary structure of the ZF was characterized by CD spectroscopy. 


\subsubsection{Fluorescence Assay}

The experiments were carried out in 20-mM Tris buffer with $50-\mathrm{mM} \mathrm{NaCl}$ at $\mathrm{pH} 7.4 .5 \mu \mathrm{M}$ of zinc finger were titrated with pure compounds at molar ratios of 1-12 (ZF to drug). The fluorescence of 12 molar equivalents of natural compounds was monitored in the same buffer. Samples were irradiated with $280 \mathrm{~nm}$ of light, and spectra were recorded from 280 to $450 \mathrm{~nm}$ with a scan rate of $600 \mathrm{~nm} / \mathrm{min}$ at $25^{\circ} \mathrm{C}$. The slit was $4.0 \mathrm{~nm}$. The emission maximum $(356 \mathrm{~nm})$ was measured after each titration. Please see Supplementary materials for more details.

\subsubsection{Mass Spectrometry}

For the mass spectrometry experiments, the zinc finger samples were prepared in an aqueous solvent at $1 \mathrm{mM}$ and incubated with an appropriate concentration of natural compounds in methanol. The reaction solutions were diluted with methanol/water (1:1 $v / v)$ to $\sim 10 \mu \mathrm{M}$. Experiments were carried out in the positive mode. Samples were directly infused at a flow rate of $1 \mu \mathrm{L} / \mathrm{min}$ using a source voltage of $3.5 \mathrm{kV}$. The source temperature was maintained at $280{ }^{\circ} \mathrm{C}$ throughout. The data was processed by Xcalibur software.

\subsubsection{Docking}

For molecular docking, AutoDock Vina software was used [39]. The NMR structure of HIV-NCp7 was downloaded from the PDB (PDB code: 1MFS). The structures of ILQ and CUR were generated by ChemDraw and OpenBabel. The charge was automatically added into ILQ and CUR, and the rotation of the bond was defined by the AutoTors program in AutoDock. Before docking, polar hydrogen atoms were added to the HIV-NCp7 protein; then, the Kollman charge was calculated. The docked grid box was a cubic 3D space with a size of $20 \AA \times 20 \AA \times 20 \AA$ and a grid interval of $1 \AA$.

\section{Conclusions}

Turmeric and licorice are widely used in traditional Chinese medicine, and they have been shown to be able to inhibit HIV. However, the detailed mechanism how is still unclear. The NCp ZF protein plays a central role in HIV virus replication, and it can stack with aromatic compounds. Since liquiritin, isoliquiritin, glycyrrhizic acid, glycyrrhetinic acid and curcumin are the major active components of licorice and turmeric, the binding between $\mathrm{NCp} 7$ and those natural compounds was studied herein. Fluorescence results showed that ILQ has the strongest binding ability among the tested compounds, followed by curcumin. The mass spectrometry analysis showed that the interaction between ILQ/CUR and the $\mathrm{NCp} 7$ zinc finger peptide was mediated by a noncovalent interaction, which was consistent with the CD spectra results. Molecule docking revealed the edge-to-face and face-to-face $\pi-\pi$ stacking between the protein tryptophan and ILQ and CUR aromatic rings. This study provided scientific evidence and hints of the antiviral activity of turmeric and licorice.

Supplementary Materials: The following are available online: Figure S1. The fluorescence emission spectra of different complexes $(30 \mu \mathrm{M})$. Figure S2. Fluorescence emission spectra of $\mathrm{ZF}$ after adding increasing amounts of LQ, ILQ, GL, GA and CUR, respectively. Figure S3. The fluorescence emission spectra of ZF upon incubation with CUR or ILQ at different time intervals. Spectra were recorded with $5-\mu \mathrm{M}$ ZF incubated with 30- $\mu \mathrm{M}$ CUR/ILQ for $10 \mathrm{~min}, 30 \mathrm{~min}, 1 \mathrm{~h}$ and $12 \mathrm{~h}$, respectively. Figure S4. ESI-MS spectra of the reaction mixture between ZF and ILQ. The selected region of the spectra showed $3+$ charged peaks. The reaction was performed on $10-\mu \mathrm{M} Z \mathrm{ZF}$ with $30-\mu \mathrm{M}$ ILQ. The spectra were recorded after incubation for $15 \mathrm{~min}(\mathrm{~A}), 2 \mathrm{~h}(\mathrm{~B})$ and $5 \mathrm{~h}(\mathrm{C})$, respectively. Figure S5. Species observed in the ESI-MS spectrum for the reaction mixture between ZF and ILQ. Observed (A,C,E) and calculated (B,D,F) mass spectra of the species. Figure S6. ESI-MS spectra of the reaction mixture between ZF and CUR. $10 \mu \mathrm{M}$ of zinc finger were incubated with $60-\mu \mathrm{M}$ CUR for $15 \mathrm{~min}$ (top). $10 \mu \mathrm{M}$ of zinc finger were incubated with $30-\mu \mathrm{M}$ CUR for $15 \mathrm{~min}$ (bottom). Figure S7. ESI-MS spectra of the reaction mixture between ZF and CUR. The selected region of the spectra showed $3+$ charged peaks. The reaction was performed on $10-\mu \mathrm{M}$ $\mathrm{ZF}$ with $60-\mu \mathrm{M}$ CUR. The spectra were recorded after incubation for $15 \mathrm{~min}, 2 \mathrm{~h}$ and $5 \mathrm{~h}$, respectively. Figure S8. The species observed in the ESI-MS spectrum for the reaction mixture between ZF and CUR. Observed (left) and calculated (right) mass spectra of the species. 
Author Contributions: Conceptualization, Z.D.; investigation, R.W. and Y.W.; methodology, P.Y.; writing-original draft preparation, R.W. and Y.W.; writing—review and editing, Z.D. and M.W.; supervision, Z.D. and H.J. and funding acquisition, Z.D. All authors have read and agreed to the published version of the manuscript.

Funding: This work was supported by the National Natural Science Foundation of China (no. 21904044 and 81874309).

Institutional Review Board Statement: Not applicable.

Informed Consent Statement: Not applicable.

Data Availability Statement: The data presented in this study are available on request from the corresponding author.

Acknowledgments: The technical help from the Analytical Centre of Huazhong University of Science and Technology is gratefully acknowledged.

Conflicts of Interest: The authors declare that there are no conflicts of interest.

Sample Availability: Samples of the compounds are available from the authors.

\begin{tabular}{ll}
\multicolumn{2}{l}{ Abbreviations } \\
ZF & zinc finger \\
LQ & liquiritin \\
ILQ & isoliquiritin \\
GL & glycyrrhizic acid \\
GA & glycyrrhetinic acid \\
CUR & curcumin \\
HIV-NCp7 & the human immunodeficiency virus nucleocapsid protein \\
NCIs & NC inhibitors \\
CD & circular dichroism \\
ESI-MS & electrospray ionization-mass spectrometer
\end{tabular}

\section{References}

1. Daniel, A.G.; Peterson, E.J.; Farrell, N.P. The bioinorganic chemistry of apoptosis: Potential inhibitory zinc binding sites in caspase-3. Angew. Chem. Int. Ed. 2014, 53, 4098-4101. [CrossRef]

2. Anzellotti, A.I.; Liu, Q.; Bloemink, M.J.; Scarsdale, J.N.; Farrell, N. Targeting retroviral Zn finger-DNA interactions: A smallmolecule approach using the electrophilic nature of trans-platinum-nucleobase compounds. Chem. Biol. 2006, 13, 539-548. [CrossRef] [PubMed]

3. Anzellotti, A.I.; Farrell, N.P. Zinc metalloproteins as medicinal targets. Chem. Soc. Rev. 2008, 37, 1629-1651. [CrossRef] [PubMed]

4. Deshmukh, L.; Tugarinov, V.; Appella, D.H.; Clore, G.M. Targeting a dark excited state of HIV-1 nucleocapsid by antiretroviral thioesters revealed by NMR spectroscopy. Angew. Chem. Int. Ed. 2018, 57, 2687-2691. [CrossRef] [PubMed]

5. Mori, M.; Kovalenko, L.; Lyonnais, S.; Antaki, D.; Torbett, B.E.; Botta, M.; Mirambeau, G.; Mely, Y. Nucleocapsid protein: A desirable target for future therapies against HIV-1. Curr. Top. Microbiol. 2015, 389, 53-92.

6. Mori, M.; Kovalenko, L.; Malancona, S.; Saladini, F.; De Forni, D.; Pires, M.; Humbert, N.; Real, E.; Botzanowski, T.; Cianferani, S.; et al. Structure-based identification of HIV-1 nucleocapsid protein inhibitors active against wild-type and drug-resistant HIV-1 strains. ACS Chem. Biol. 2018, 13, 253-266. [CrossRef]

7. Tantillo, C.; Ding, J.; Jacobo-Molina, A.; Nanni, R.G.; Boyer, P.L.; Hughes, S.H.; Pauwels, R.; Andries, K.; Janssen, P.A.; Arnold, E. Locations of anti-aids drug binding sites and resistance mutations in the three-dimensional structure of HIV-1 reverse transcriptase: Implications for mechanisms of drug inhibition and resistance. J. Mol. Biol. 1994, 243, 369-387. [CrossRef]

8. Remy, E.; de Rocquigny, H.; Petitjean, P.; Muriaux, D.; Theilleux, V.; Paoletti, J.; Roques, B.P. The annealing of tRNA3Lys to human immunodeficiency virus type 1 primer binding site is critically dependent on the ncp7 zinc fingers structure. J. Biol. Chem. 1998, 273, 4819-4822. [CrossRef]

9. De Rocquigny, H.; Delaunay, T.; Petitjean, P.; Fournié-Zaluski, M.; Roques, B. La structure native de la ncp7 d'HIV-1 est nécessaire pour la reconnaissance spécifique de la séquence d'encapsidation psi de l'arn genomique: Etude par résonance plasmonique de surface. Regard. Biochim. 1998, 2, 44-50.

10. Bailly, F.; Cotelle, P. Anti-HIV activities of natural antioxidant caffeic acid derivatives: Toward an antiviral supplementation diet. Curr. Med. Chem. 2005, 12, 1811-1818. [CrossRef] 
11. Singh, I.P.; Bodiwala, H.S. Recent advances in anti-HIV natural products. Nat. Prod. Rep. 2010, 27, 1781-1800. [CrossRef]

12. Li, Z.J.; Zhao, Y.R.; Lin, W.W.; Ye, M.; Ling, X.M. Rapid screening and identification of active ingredients in licorice extract interacting with v3 loop region of HIV-1 gp120 using ace and ce-ms. J. Pharm. Biomed. Anal. 2015, 111, 28-35. [CrossRef]

13. Huang, M.L.; Cheng, Z.Z.; Wang, L.; Feng, Y.L.; Huang, J.G.; Du, Z.F.; Jiang, H.L. A targeted strategy to identify untargeted metabolites from in vitro to in vivo: Rapid and sensitive metabolites profiling of licorice in rats using ultra-high performance liquid chromatography coupled with triple quadrupole-linear ion trap mass spectrometry. J. Chromatogr. B 2018, 1092, 40-50.

14. Watanbe, H.; Miyaji, C.; Makino, M.; Abo, T. Therapeutic effects of glycyrrhizin in mice infected with lp-bm5 murine retrovirus and mechanisms involved in the prevention of disease progression. Biotherapy 1996, 9, 209-220. [CrossRef] [PubMed]

15. Ito, M.; Nakashima, H.; Baba, M.; Pauwels, R.; De Clercq, E.; Shigeta, S.; Yamamoto, N. Inhibitory effect of glycyrrhizin on the in vitro infectivity and cytopathic activity of the human immunodeficiency virus [HIV (HTLV-III/LAV)]. Antivir. Res. 1987, 3, 127-137. [CrossRef]

16. Mori, K.; Sakai, H.; Suzuki, S.; Akutsu, Y.; Ishikawa, M.; Imaizumi, M.; Tada, K.; Aihara, M.; Sawada, Y.; Yokoyama, M. Effects of glycyrrhizin (SNMC: Stronger neo-minophagen c) in hemophilia patients with HIV-1 infection. Tohoku J. Exp. Med. 1990, 162, 183-193. [CrossRef] [PubMed]

17. Abhijit, M.; Krishnamachari, R.; John, W.; Kurt, W.K.; Yves, P. Inhibition of human immunodeficiency virus type-1 integrase by curcumin. Biochem. Pharmacol. 1995, 49, 1165-1170.

18. Jennings Morgan, R.; Parks Robin, J. Curcumin as an antiviral agent. Viruses 2020, 6, 3412-3419. [CrossRef]

19. Prasad, S.; Tyagi Amit, K. Curcumin and its analogues: A potential natural compound against HIV infection and aids. Food Funct. 2015, 12, 1242. [CrossRef]

20. Sergei, N.; Charlee, M.; Evaristus, N.; Xionghao, L.; MariaMananita, H.; Amol, K.; Andrey, I.; Namita, K.; Tatiana, A. Inhibition of HIV-1 by curcumin a, a novel curcumin analog. Drug Des. Dev. Ther. 2015, 9, 5051-5060.

21. Sui, Z.; Salto, R.; Li, J.; Craik, C.; Ortiz de Montellano Paul, R. Inhibition of the HIV-1 and HIV-2 proteases by curcumin and curcumin boron complexes. Bioorg. Med. Chem. 1993, 1, 415-422. [CrossRef]

22. James, J.S. Curcumin: Clinical trial finds no antiviral effect. AIDS Treat. News 1996, 2, 1-2.

23. Rodrigues, T.; Reker, D.; Schneider, P.; Schneider, G. Counting on natural products for drug design. Nat. Chem. 2016, 8, 531-541. [CrossRef] [PubMed]

24. Qin, X.J.; Feng, M.Y.; Liu, H.; Ni, W.; Rauwolf, T.; Porco, J.A.; Yan, H.; He, L.; Liu, H.Y. Eucalyptusdimers a-c, dimeric phloroglucinol phellandrene meroterpenoids from eucalyptus robusta. Org. Lett. 2018, 20, 5066-5070. [CrossRef] [PubMed]

25. Liu, Y.; Chen, G.; Chen, X.; Chen, S.-X.; Gan, L.-S.; Yuan, T. Colocynthenins a-d, ring-a seco-cucurbitane triterpenoids from the fruits of citrullus colocynthis. J. Nat. Prod. 2018, 81, 2115-2119. [CrossRef]

26. Hofstadler, S.A.; Sannes-Lowery, K.A. Applications of ESI-MS in drug discovery: Interrogation of noncovalent complexes. Nat. Rev. Drug Discov. 2006, 5, 585-595. [CrossRef]

27. Haris, P.I. Can infrared spectroscopy provide information on protein-protein interactions? Biochem. Soc. Trans. 2010, 38, 940-946. [CrossRef] [PubMed]

28. Karthick, T.; Balachandran, V.; Perumal, S. Spectroscopic investigations, molecular interactions, and molecular docking studies on the potential inhibitor "thiophene-2-carboxylicacid". Spectrochim. Acta A 2015, 141, 104-112. [CrossRef]

29. Sirajuddin, M.; Ali, S.; Badshah, A. Drug-DNA interactions and their study by uv-visible, fluorescence spectroscopies and cyclic voltametry. J. Photochem. Photobiol. B 2013, 124, 1-19. [CrossRef]

30. Stephen, A.G.; Worthy, K.M.; Towler, E.; Mikovits, J.A.; Sei, S.; Roberts, P.; Yang, Q.E.; Akee, R.K.; Klausmeyer, P.; McCloud, T.G.; et al. Identification of HIV-1 nucleocapsid protein: Nucleic acid antagonists with cellular anti-HIV activity. Biochem. Biophys. Res. Commun. 2002, 296, 1228-1237. [CrossRef]

31. De Paula, Q.A.; Mangrum, J.B.; Farrell, N.P. Zinc finger proteins as templates for metal ion exchange: Substitution effects on the c-finger of HIV nucleocapsid ncp7 using $\mathrm{m}$ (chelate) species ( $\mathrm{m}=\mathrm{pt}$, pd, au). J. Inorg. Biochem. 2009, 103, 1347-1354. [CrossRef] [PubMed]

32. Tsotsoros, S.D.; Lutz, P.B.; Daniel, A.G.; Peterson, E.J.; de Paiva, R.E.F.; Rivera, E.; Qu, Y.; Bayse, C.A.; Farrell, N.P. Enhancement of the physicochemical properties of [pt(dien)(nucleobase)](2+) for HIV-NCp7 targeting. Chem. Sci. 2017, 8, 1269-1281. [CrossRef] [PubMed]

33. Kawai, H.; Tarui, M.; Doi, M.; Ishida, T. Enhancement of aromatic amino acid-nucleic acid base stacking interaction by metal coordination to base: Fluorescence study on a tryptophan-pt (ii)-guanine ternary complex. FEBS Lett. 1995, 370, 193-196. [CrossRef]

34. Jinxia, D.; James, A.K.; Joseph, J.B.; Tino, S.; Raveendra, D.; Yves, P.; Nouri, N. Mining the NCI antiviral compounds for HIV-1 integrase inhibitors. Bioorg. Med. Chem. 2006, 14, 3785-3792.

35. Sheng, Y.P.; Cao, K.M.; Li, J.; Hou, Z.H.; Yuan, S.M.; Huang, G.M.; Liu, H.K.; Liu, Y.Z. Selective targeting of the zinc finger domain of HIV nucleocapsid protein ncp7 with ruthenium complexes. Chem. Eur. J. 2018, 24, 19146-19151. [CrossRef]

36. Demicheli, C.; Frezard, F.; Mangrum, J.B.; Farrell, N.P. Interaction of trivalent antimony with a cchc zinc finger domain: Potential relevance to the mechanism of action of antimonial drugs. Chem. Commun. 2008, 39, 4828-4830. [CrossRef]

37. Abbehausen, C.; Peterson, E.J.; de Paiva, R.E.F.; Corbi, P.P.; Formiga, A.L.B.; Qu, Y.; Farrell, N.P. Gold(i)-phosphine-n-heterocycles: Biological activity and specific (ligand) interactions on the C-terminal HIV-NCp7 zinc finger. Inorg. Chem. 2013, 52, 11280-11287. [CrossRef] [PubMed] 
38. Quintal, S.M.; de Paula, Q.A.; Farrell, N.P. Zinc finger proteins as templates for metal ion exchange and ligand reactivity. Chemical and biological consequences. Metallomics 2011, 3, 121-139. [CrossRef]

39. Trott, O.; Olson, A.J. AutoDock Vina: Improving the speed and accuracy of docking with a new scoring function, efficient optimization, and multithreading. J. Comput. Chem. 2010, 31, 455-461. [CrossRef] 